\title{
Combined computational and experimental investigation of the refractory properties of $\mathrm{La}_{2} \mathrm{Zr}_{2} \mathrm{O}_{7}$
}

\author{
Qi-Jun Honga,b,*, Sergey V. Ushakov ${ }^{\mathrm{c}}$, Alexandra Navrotsky ${ }^{\mathrm{c}}$, Axel van de \\ Walle $^{\mathrm{b}}$ \\ ${ }^{a}$ Division of Chemistry and Chemical Engineering, California Institute of Technology, \\ Pasadena, California 91125, USA \\ ${ }^{b}$ School of Engineering, Brown University, Providence, Rhode Island 02912, USA \\ ${ }^{c}$ Peter A. Rock Thermochemistry Laboratory and NEAT ORU (Nanomaterials in the \\ Environment, Agriculture, and Technology Organized Research Unit), University of \\ California, Davis, One Shields Avenue, Davis, California 95616, USA
}

\begin{abstract}
We demonstrate how key material properties that provide guidance in the design of refractory materials can be accurately determined via $a b$ initio thermodynamic calculations in conjunction with experimental techniques based on synchrotron X-ray diffraction and thermal analysis under laser-heated aerodynamic levitation. The properties considered include melting point, heat of fusion, heat capacity, thermal expansion coefficients, thermal stability and sublattice disordering, as illustrated in a motivating example of lanthanum zirconate $\left(\mathrm{La}_{2} \mathrm{Zr}_{2} \mathrm{O}_{7}\right)$. This work also sheds light on the unresolved controversy of possible phase transition before melting and identifies specific mechanisms responsible for the material's high melting point. This study benefits from the use of two very recent techniques: (i) a new small-cell coexistence method that enables the accurate and efficient determination of the melting points from ab initio calculations alone; (ii) the experimental determination of solid structure at high temperatures by high temperature synchrotron X-ray diffraction on laser heated aerodynamically levitated samples.
\end{abstract}

Keywords: lanthanum zirconate, heat of fusion, melting temperature, disorder, thermal expansion and heat capacity

\footnotetext{
* Corresponding author

Email address: qhong@caltech.edu (Qi-Jun Hong)
}

Preprint submitted to Acta Materialia

October 2, 2014 


\section{Introduction}

Lanthanum zirconate is being investigated for a wide range of applications, including solid oxide fuel cell electrodes [1, 2], fluorescence screens [3, 4], catalysts [5, 6] and high-temperature superconducting coated conductors [7, 8]. In 5 particular, $\mathrm{La}_{2} \mathrm{Zr}_{2} \mathrm{O}_{7}$ is proposed as a promising thermal-barrier coating (TBC) material 9 , 10, 11, 12, 13, 14, 15, 16, 17, 18, to replace the most-commonly used yttria-stabilized zirconia (YSZ), for the following reasons: (i) $\mathrm{La}_{2} \mathrm{Zr}_{2} \mathrm{O}_{7}$ coating has high phase stability against thermal treatment, thus it is capable to function under higher temperatures, far beyond the limited operation temperature $\left(1200{ }^{\circ} \mathrm{C}\right.$ ) of $\mathrm{YSZ}\left[19\right.$; (ii) $\mathrm{La}_{2} \mathrm{Zr}_{2} \mathrm{O}_{7}$ has a lower thermal conductivity, which provides better thermal insulation for the components from large and prolonged heat loads; (iii) $\mathrm{La}_{2} \mathrm{Zr}_{2} \mathrm{O}_{7}$ has a lower oxygen ion diffusivity (less oxygen-transparent), so it protects the body coat from oxidation. The major drawback of $\mathrm{La}_{2} \mathrm{Zr}_{2} \mathrm{O}_{7}$ as a TBC coating is its small thermal expansion coefficient, which does not match that of the superalloy in the body coat.

The assessment of novel refractory materials, such as lanthanum zirconate, demands the determination of a number of physical, chemical and mechanical properties. We demonstrate how ab initio calculations can be used in conjunction with advanced experimental techniques (synchrotron X-ray diffraction on laser heated aerodynamically levitated samples and thermal analysis above $2000{ }^{\circ} \mathrm{C}$ ) to provide a more complete and accurate picture of thermal properties. Our combined computational and experimental investigation determines key material properties, ranging from enthalpy of fusion, melting temperature, to thermal expansion and heat capacity. We also investigate the possibility of high-temperature sublattice disordering.

Some properties are more directly obtained via computational means. For instance, the heat of fusion, the heat capacity and the thermal expansion are straightforward to calculate, but clearly benefit from an experimental verification to ascertain the validity of the modeling assumptions (e.g., stable structure, 
sources of entropy and the reliance on density functional theory). These measurements, while simple at low temperature, become challenging above $1500{ }^{\circ} \mathrm{C}$, as considered in this work, because they necessitate development of new experimental techniques and calibration standards. In contrast, other properties are more easily accessible experimentally. The most striking example is the melt-

35 ing point, where computational efforts via ab initio methods demand advanced techniques to reduce the computational burden $20,21,22,23,24,25,26,27,28$. In this work, the recently proposed small-cell coexistence method [29] makes it feasible to calculate the melting point of a compound with a substantially more complex crystal structure than considered in prior ab initio computational stud-

40 ies. Given the challenges associated with these calculations, experimental validation is particularly important. Another important example of computationalexperimental complementarity is the determination of the presence of sublattice disordering. While it is difficult to assess the state of order of the oxygen sublattice via X-ray diffraction (due to its small scattering factor), our computations can rather clearly establish the possibility of such a disordering transition since oxygen diffusion is visible in our simulations. Conversely, disordering on the cation sublattice is not as easily accessible in our simulations but would have been detected experimentally if it were present.

Overall, our results show very good agreement between experimental measurements and their computational counterparts, even in cases reaching the limit of today's computational or experimental methods. This situation supports the validity of our thermodynamic description.

This paper is organized as follows. The detailed computational and experimental approaches are first described in Section 2, Results and discussions are presented in Section 3 .

\section{Methods}

Pyrochlore $\mathrm{La}_{2} \mathrm{Zr}_{2} \mathrm{O}_{7}$ structure has the space group of $F d \overline{3} m$ that derives from the fluorite-like arrangement of atoms. The cubic pyrochlore structure of 
$\mathrm{La}_{2} \mathrm{Zr}_{2} \mathrm{O}_{7}$ has been discussed in detail [30, 31, 32, 33]. As shown in Fig. 1 .

the $\mathrm{La}^{3+}$ ions occupy the special site $16 d(1 / 2,1 / 2,1 / 2)$ and the $\mathrm{Zr}^{4+}$ ions occupy the special site $16 c(0,0,0)$. The oxygen atoms are located at sites $48 f$ $(x, 1 / 8,1 / 8)$ and $8 b(3 / 8,3 / 8,3 / 8)$, and the oxygen vacancies at $8 a(1 / 8,1 / 8,1 / 8)$. Table 1 lists the lattice parameter and the value of $x$ from both calculation and experiment. The primitive unit of $\mathrm{La}_{2} \mathrm{Zr}_{2} \mathrm{O}_{7}$ consists of $4 \mathrm{La}, 4 \mathrm{Zr}$ and $14 \mathrm{O}$ atoms. There are 88 atoms in a face-centered cubic (fcc) conventional unit cell. $\mathrm{La}_{2} \mathrm{Zr}_{2} \mathrm{O}_{7}$ is also reported in the defect fluorite $(F m \overline{3} m)$ structure as transitory during radiation damage and from low temperature synthesis. The defect fluorite structure can be derived from pyrochlore by disordering on both cation and anion sublattices.

\subsection{Computational}

We employ first-principles density functional theory [34, 35, 36] to model $\mathrm{La}_{2} \mathrm{Zr}_{2} \mathrm{O}_{7}$. All electronic structures are calculated by the Vienna Ab-initio Simulation Package (VASP) [37, 38, 39, with the projector-augmented-wave (PAW) [40] implementation and the generalized gradient approximation (GGA)

75 for exchange-correlation energy, in the form known as Perdew-Burke-Ernzerhof (PBE) 41. For Lanthanum, the inner core $5 s$ and $5 p$ electrons are relaxed for

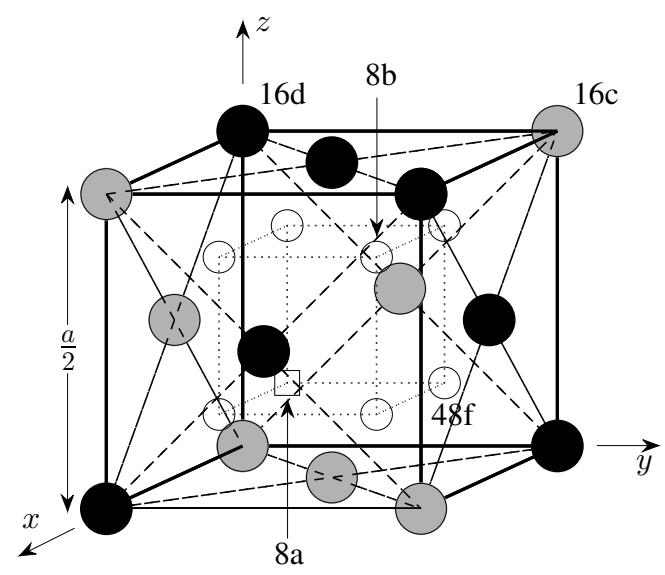

Figure 1: Pyrochlore structure of $\mathrm{La}_{2} \mathrm{Zr}_{2} \mathrm{O}_{7}$. 
electronic structure optimization. The electronic temperature is accounted for by imposing a Fermi distribution of the electrons on the energy level density of states, so it is consistent with the ionic temperature.

First-principles molecular dynamics (MD) techniques are utilized to simulate atomic movements and trajectories of $\mathrm{La}_{2} \mathrm{Zr}_{2} \mathrm{O}_{7}$, in order to compute various properties. Specifically, MD simulations are carried out under constant number of atoms, pressure and temperature condition (NPT, isothermal-isobaric ensemble). Here the thermostat is conducted under the Nose-Hoover chain formalism [42, 43, 44, 45. The barostat is realized by adjusting volume every 200 steps according to average pressure. Although this does not formally generate an isobaric ensemble, this approach has been shown to provide an effective way to change volume smoothly and to avoid the unphysical large oscillation caused by commonly used barostats [29]. The $\mathrm{La}_{2} \mathrm{Zr}_{2} \mathrm{O}_{7}$ pyrochlore structure is taken 90 as a starting point for all MD simulations of the solid phase.

\subsection{Experimental}

Laser melting of $\mathrm{La}_{2} \mathrm{Zr}_{2} \mathrm{O}_{7}$ for diffraction and thermal analysis experiments causes some loss of La resulting in $\mathrm{La}_{1.96} \mathrm{Zr}_{2.03} \mathrm{O}_{7}$ stoichiometry, as determined by microprobe analysis. Thermal analysis is performed in sealed tungsten crucibles in Setaram Setsys 2400 instrument modified to enable sample temperature monitoring with spectropyrometer [46, 47]. High temperature X-ray diffraction experiments are performed on an aerodynamic levitator at beamline 11-ID-C at the Advanced Photon Source, Argonne National Laboratory. A detailed description of experimental setups is published elsewhere [48. Processing of diffraction

Table 1: Comparison between experimental and calculated data of $\mathrm{La}_{2} \mathrm{Zr}_{2} \mathrm{O}_{7}$.

$\begin{array}{ccccc}\text { method } & \text { Expt. } & \text { PBE } & \text { HSE } & \text { HF } \\ a(\AA) & 10.805 & 10.880 & 10.833 & 10.728 \\ x & 0.4200 & 0.4172 & 0.4171 & 0.4170\end{array}$




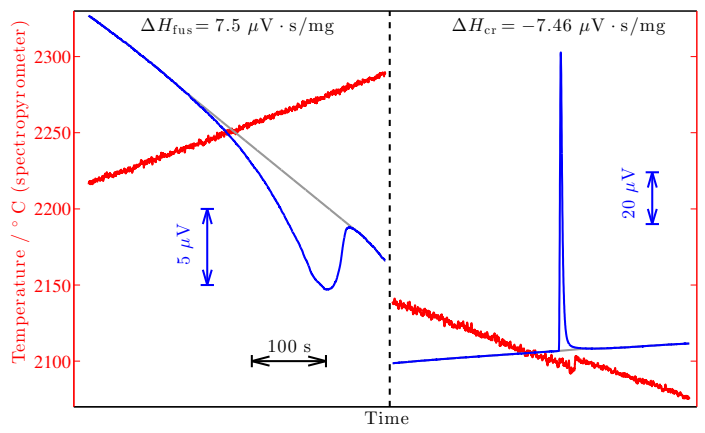

Figure 2: Melting and crystallization of $\mathrm{La}_{1.96} \mathrm{Zr}_{2.03} \mathrm{O}_{7}$ in thermal analyzer at $10{ }^{\circ} \mathrm{C} / \mathrm{min}$ heating/cooling rate. $150^{\circ} \mathrm{C}$ undercooling is evident. Integration of peaks in heat flow (blue trace) on melting and crystallization gives similar values indicating direct crystallization of pyrochlore phase.

zirconate melt is performed in UC Davis using custom built splittable nozzle aerodynamic levitator with $\mathrm{CO}_{2}$ laser heating.

\section{Results and discussions}

\subsection{Fusion enthalpy}

Fusion enthalpy is calculated as the enthalpy difference between the solid and the liquid at $2500 \mathrm{~K}$. We perform NPT MD simulation to generate a trajectory for each phase. The enthalpy is evaluated as an average over time during the MD trajectory.

We use a supercell containing 88 atoms $\left(8 \mathrm{La}_{2} \mathrm{Zr}_{2} \mathrm{O}_{7}\right)$ for the pyrochlore phase. Modeling liquid phase on a periodic cell is subject to relatively stronger finite-size effect, since the liquid is imposed by the unrealistic periodic boundary condition. So we choose a larger cell with 176 atoms to describe the liquid phase more accurately.

We perform further correction on the data from PBE to a more accurate hybrid functional of Heyd-Scuseria-Ernzerhof (HSE) [50]. For both the pyrochlore and liquid phases, we compute energy differences between PBE and HSE on randomly chosen snapshots from MD trajectories, and then combine them as a correction on the fusion enthalpy. 
The fusion enthalpy is calculated to be $0.26 \mathrm{eV} /$ atom $(270 \mathrm{~kJ} / \mathrm{mol})$. After HSE correction, it is $0.28 \mathrm{eV} /$ atom $(300 \mathrm{~kJ} / \mathrm{mol})$. The major error is considered to be the finite-size effect from the liquid phase. Based on study on different system size (88 atoms versus 176 atoms), the error is estimated to be 0.02 $\mathrm{eV} /$ atom $(20 \mathrm{~kJ} / \mathrm{mol})$.

First experimental measurement of fusion enthalpy for $\mathrm{La}_{2} \mathrm{Zr}_{2} \mathrm{O}_{7}$ pyrochlore was reported by Radha [17. New set of measurements (Fig. 22) is performed on laser melted $\mathrm{La}_{1.96} \mathrm{Zr}_{2.03} \mathrm{O}_{7}$ in sealed tungsten crucibles to prevent carbon contamination from furnace. Uncertainty in experimental measurements of fusion enthalpy by thermal analysis is difficult to evaluate due to the absence of enthalpy calibration standard above $\mathrm{Al}_{2} \mathrm{O}_{3}$ melting temperature $\left(2054{ }^{\circ} \mathrm{C}\right)$. The value $300 \pm 50 \mathrm{~kJ} / \mathrm{mol}$ can be taken as a conservative evaluation of both sets of experimental measurements.

The close agreement between computation and experiment strongly supports the validity of the results. We find the computational approach relatively easier than its experimental counterpart in determining heat of fusion, when temperature reaches far beyond the $\mathrm{Al}_{2} \mathrm{O}_{3}$ melting temperature $\left(2054{ }^{\circ} \mathrm{C}\right)$. Enthalpy calibration standard for higher temperature, e.g., $\mathrm{Y}_{2} \mathrm{O}_{3}\left(2430{ }^{\circ} \mathrm{C}\right)$, would increase the accuracy of fusion enthalpy measurements by commercially available thermal analyzers. We plan to work on it in the next step, using a combined experimental and computational approach.

\subsection{Melting temperature}

We employ our small-size coexistence method to calculate the melting temperatures. The result is shown in Fig. 3. The details of this method have been described previously in Ref. [29], and here we briefly reiterate it. While traditional two-phase coexistence method accurately predicts melting temperature based on stabilized coexistence, it is computationally prohibitive in the DFT framework, due to large system size (usually at least 1,000 atoms) to stabilize the interface. We reduce the cell size down to about 100 atoms, so it becomes feasible to combine directly with DFT. Though coexistence can no longer be 
stabilized in a small cell, we manage to obtain the melting temperature based on the statistical distribution of the final phases, i.e., we run multiple duplicates of small-cell coexistences to evaluate the probabilities of the final phases. Starting from solid-liquid coexistence, we monitor the system evolution until it reaches a pure final phase, either a solid or a liquid, and we calculate such probabilities based on the results from multiple duplicated simulations. We then fit the melting temperature from these probability distributions. It can be formally derived that the distribution, at a particular temperature, is

$$
\frac{N_{\text {liquid }}}{N_{\text {solid }}}=\exp \left(-\beta G^{l-s} l_{x} / 2\right),
$$

where $N_{\text {liquid }} / N_{\text {solid }}$ is the ratio of the number of MD runs that coexistence ends in either liquid or solid, $G^{l-s}$ is the Gibbs free energy difference at temperature $T, l_{x}$ is a finite-size parameter and $\beta$ is $1 / k_{\mathrm{B}} T$. The Gibbs free energy difference is

$$
\begin{gathered}
H^{s}(T)=H^{s}\left(T_{m}\right)+C_{p}^{s}\left(T-T_{m}\right) \\
H^{l-s}(T)=H^{l-s}\left(T_{m}\right)+C_{p}^{l-s}\left(T-T_{m}\right) \\
G^{l-s}(T)=\frac{T_{m}-T}{T_{m}} H^{l-s}\left(T_{m}\right)-C_{p}^{l-s} \frac{\left(T-T_{m}\right)^{2}}{T_{m}} .
\end{gathered}
$$

where $T_{m}$ is melting temperature, $H$ is enthalpy, $H^{l-s}$ is heat of fusion, $C_{p}$ is heat capacity. In Ref. [29], we validated this relation through various examples

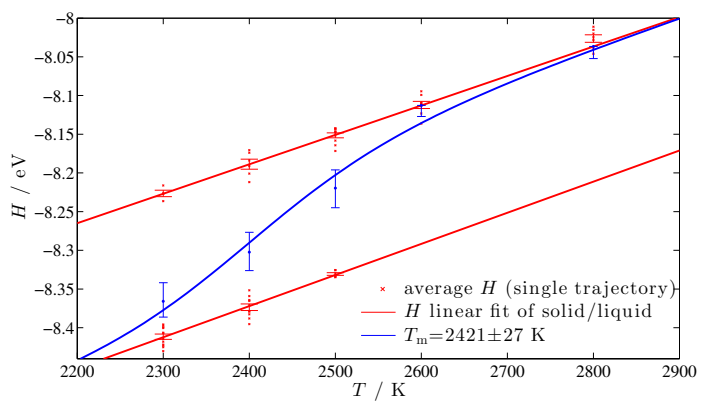

Figure 3: The melting temperature of $\mathrm{La}_{2} \mathrm{Zr}_{2} \mathrm{O}_{7}$ fitted according to Eqs. (1) to (4). 
Table 2: Details of HSE correction on the melting temperature.

$$
\begin{array}{cc}
T_{m}^{\mathrm{PBE}} & 2420 \mathrm{~K} \\
\Delta \mu^{s} & -2.634 \mathrm{eV} \\
\Delta \mu^{l} & -2.610 \mathrm{eV} \\
\Delta H^{l-s} & 0.28 \mathrm{eV} \\
\Delta T_{m} & 210 \mathrm{~K} \\
T_{m}^{\mathrm{HSE}} & 2630 \mathrm{~K}
\end{array}
$$

from empirical potentials. Finite size effect was studied systematically. We found we can usually control the error to be below $100 \mathrm{~K}$, using a cell containing around 100 atoms.

The simulations on $\mathrm{La}_{2} \mathrm{Zr}_{2} \mathrm{O}_{7}$ are performed on a $1 \times 1 \times 2$ fcc supercell, which contains 176 atoms and measures approximately $11 \times 11 \times 22 \AA^{3}$. The plane wave energy cutoff is set to $400 \mathrm{eV}$ and it is further increased to $500 \mathrm{eV}$ for pressure correction. NPT MD simulations are carried out to trace the evolution of coexisting systems. Only $\Gamma$-point is used throughout the calculations. To estimate the error of using this single k-point, we compare with fully converged k-point grid and compute energy difference on randomly chosen configurations from both solid and liquid. The root mean square error is less than $1 \mathrm{meV} /$ atom.

The melting temperature $T_{m}$ is calculated to be $2420 \pm 28 \mathrm{~K}$, as shown in Fig. 3. We apply a correction based on the more accurate hybrid functional of HSE. First, free energy correction is made on each phase following the thermodynamic integration method.

$$
\mu_{\beta}=\mu_{\alpha}+\frac{1}{N} \int_{0}^{1}\left\langle H_{\beta}-H_{\alpha}\right\rangle_{H_{\lambda}} \mathrm{d} \lambda,
$$

where $\beta$ is HSE, $\alpha$ is PBE. Due to the high computational cost of HSE, only $\lambda=0$ is used for integration. Then the melting temperature correction is evaluated as

$$
\Delta T_{m}=\left(\Delta \mu^{l}-\Delta \mu^{s}\right) \cdot \frac{T_{m}}{\Delta H^{l-s}},
$$

where $\Delta \mu^{l / s}$ are the corrections in free energies for the liquid and the solid, ${ }_{155} \Delta H^{l-s}$ is heat of fusion, and $\Delta T_{m}$ is melting temperature correction. Detailed 
Table 3: Comparison of the computational and experimental data for the melting temperature of $\mathrm{La}_{2} \mathrm{Zr}_{2} \mathrm{O}_{7}$.

$\begin{array}{lc}\text { T (C) } & \text { Reference } \\ 2150 & \text { this work, PBE } \\ 2360 & \text { this work, HSE } \\ 2260 & \text { this work, expt. } \\ 2295 & \text { Radha (2009) } 17 \\ 2340 & \text { Lakiza (2005) } 51 \\ 2230 & \text { Zoz (1978) } 52 \\ 2160 & \text { Portnoi (1972) } 53 \\ 2280 & \text { Rouanet (1971) } 54 \\ 2250 & \text { Lin (1964) } 55\end{array}$

data of the HSE correction on melting temperature are listed in Table 2, The melting temperature after HSE correction is $2630 \mathrm{~K}$.

Experiment measures the melting temperature as $2260{ }^{\circ} \mathrm{C}(2530 \mathrm{~K})$, which lies in the middle of the two theoretical predictions. Our results are in close agreement with others in the literature, as shown in Table 3

Our calculations enable us to identify the factors that contribute to a high melting point in $\mathrm{La}_{2} \mathrm{Zr}_{2} \mathrm{O}_{7}$. It has a large fusion enthalpy of $0.28 \mathrm{eV} /$ atom, which is a major contributor to the high melting point. Pyrochlore phase $\mathrm{La}_{2} \mathrm{Zr}_{2} \mathrm{O}_{7}$ is further stabilized by its high entropy, in the following two respects. An interesting behavior of the pyrochlore structure is the formation of soft vibrational modes upon thermal expansion. As shown in Fig. 4, thermal expansion not only softens the certain zone-center phonon modes, but also creates a new wide double-well feature, which significantly increases the accessible phase space. The high entropy of the solid phase also comes from diffusion of oxygen atoms. As 170 discussed in Section 3.3.1, the corresponding barrier is relatively low, so oxygen atoms visit their neighboring vacancies frequently. The likelihood to form an oxygen-disordered sublattice greatly increases the configurational entropy, which further stabilizes the solid phase. 


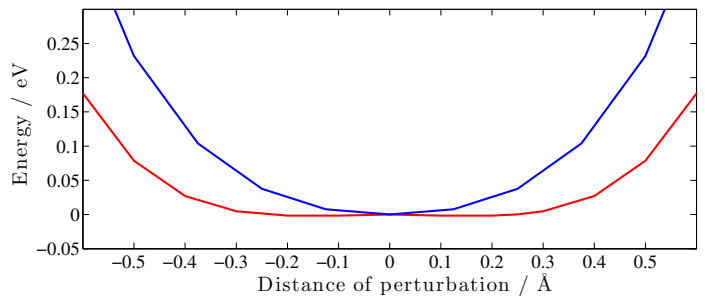

Figure 4: Potential energy upon perturbation along a zone-center soft phonon mode predominantly involving La motion along $\langle 123\rangle$ and $\langle 110\rangle$ directions (blue: $0 \mathrm{~K}$; red: $2 \%$ thermal expansion). The potential well not only softens, but also forms double-wells (the curvature at the center turns negative after the thermal expansion). This feature significantly increases the accessible phase space, which increases entropy as a result.

\subsection{Disordering} at any temperature.

Disordering has a strong impact on thermal performance of TBC materials. 
The disordering of TBC leads to volume change and hence mismatch between

body coat and topcoat, which results in cracking in TBC, a common mode of materials failure. Also, oxygen sublattice disorder determines whether a material is oxygen-transparent. If oxygen atoms can diffuse readily in TBC, the underlying body coat is exposed to the oxygen environment in the heat flux, which rapidly oxidizes the body coat and forms thermally grown oxide (TGO).

200 Irregularly grown TGO exaggerates the strain in the coating, which is a major mechanism of thermal failure.

In our simulations, we find strong evidence that the system undergoes a sublattice disordering transition (on the oxygen sublattice) just before melting. Our results also indicate that disordering of the cation sublattice is unlikely. Syn-

205 chrotron X-ray diffraction confirms cation ordering in pyrochlore up to complete melting.

The energy scale of the oxygen-sublattice disordering transition is found to be very small, hence the precise nature of the ordering in the solid phase turns out to have little impact on the calculated melting point. We find that disordering on the oxygen sublattice fortunately occurs only close to the melting point, so it will not affect the prospective use of $\mathrm{La}_{2} \mathrm{Zr}_{2} \mathrm{O}_{7}$ as thermal barrier coating since service temperatures normally do not exceed $2 / 3$ of the melting temperature of the material.

\subsubsection{Oxygen sublattice}

215 We observe oxygen diffusion in MD simulation of pyrochlore lanthanum zirconate, as shown in Fig. 5(a), which implies disordering on the oxygen sublattice. Transition state analysis also supports this finding. For oxygen moving to a nearby vacancy, the transition barrier is $0.5 \mathrm{eV}(48 \mathrm{~kJ})$, and the energy penalty for the final state after diffusion is only $0.4 \mathrm{eV}(39 \mathrm{~kJ})$, as shown in 220 Fig. 5(b). At $2500 \mathrm{~K}$, this corresponds to a Boltzmann distribution factor of 0.16 , which is further increased to $\sim 1$ since each vacancy has six degenerate neighboring oxygen atoms. These two observations strongly suggest disordering on the oxygen sublattice. 


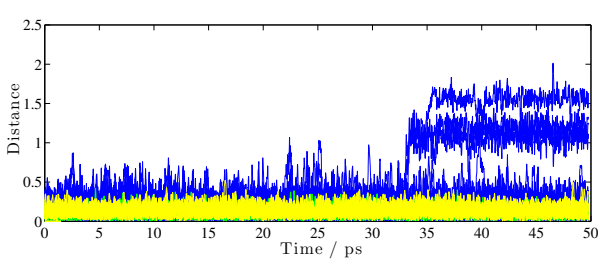

(a)

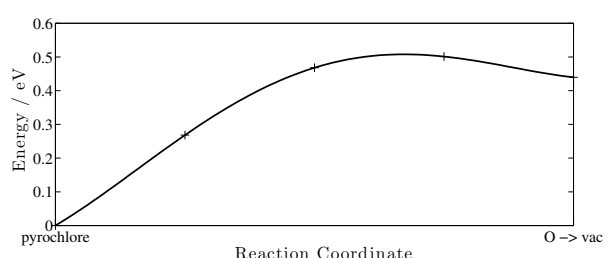

(b)

Figure 5: (a) Distance to ideal pyrochlore position during MD simulation of the solid phase at 2500 K. (blue: O; green: Zr; yellow: La.) Distance is normalized so that the nearest neighbor distance between two oxygen atoms is 1 . Diffusion is observed on two oxygen atoms near 35 ps. (b) Potential energy diagram based on nudged elastic band (NEB) method 65 66. For an oxygen atom moving to its neighboring vacancy, the activation energy is $0.5 \mathrm{eV}(48 \mathrm{~kJ})$. The energy of the final state is $0.4 \mathrm{eV}(39 \mathrm{~kJ})$ higher than pyrochlore.

A rigorous evaluation of the phase stability requires a cluster expansion [67, 68] in order to capture all important configurational states that contribute to the disordered phase. This would be very difficult to carry out for a complex ionic substance such as $\mathrm{La}_{2} \mathrm{Zr}_{2} \mathrm{O}_{7}$, especially given that the anharmonic effects are likely to be large. Instead, we employ the special quasirandom structure (SQS) approach 69] implemented in the "mcsqs" 70] code in ATAT [71] to estimate the phase stability. At first, a completely random oxygen-vacancy sublattice is studied. But we find most SQS's are unstable. Upon structural optimization, some oxygen atoms move to other lattice sites, which changes the original configuration. In particular, the tetrahedron center of $4 \mathrm{Zr}$ atoms (called 8a-site) is highly unfavored for the oxygen atom, and the high oxygen concentration at these 8a-sites is the major reason for the instability. Indeed, these sites are vacant in the pyrochlore structure. In response to this phenomenon, we limit the oxygen concentration on these 8a-sites and study a disordered phase as described below.

1. The cations are ordered as in pyrochlore.

2. Oxygen is randomly distributed on 8a-sites, with a concentration of $1 / 4$.

3. Oxygen is randomly distributed on the rest sublattice, with a concentration of $27 / 28$ (so that the chemical composition is $\mathrm{La}_{2} \mathrm{Zr}_{2} \mathrm{O}_{7}$ ). 
We generate a SQS representation of this disordered phase. The SQS is stable. With respect to pyrochlore, its energy is $33 \mathrm{meV} /$ atom $\left(35 \mathrm{~kJ} / \mathrm{mol} \mathrm{La} 2 \mathrm{Zr}_{2} \mathrm{O}_{7}\right.$ ). The entropic free energy at $2500 \mathrm{~K}$ is

$$
-T S_{\text {fluorite }}=k T \sum n_{i} \ln x_{i} / \sum n_{i}=-32 \mathrm{meV} / \text { atom. }
$$

Combining energy and entropy, this disordered phase is of comparable stability as the pyrochlore phase.

The actual disordered phase in reality will populate configurational states according to a Boltzmann distribution, rather than the uniform distribution we assume in the random structure. A Boltzmann distribution will result in the lowest free energy compared to any other distribution, therefore the free energy we calculate above is the upper limit for the disordered phase. The actual disordered phase in reality will be more stable than the randomly disordered one, which makes it very likely to replace the pyrochlore phase at high temperatures. The fact that energetic and entropic effects are so close suggests the transition temperature will be close to $2500 \mathrm{~K}$, very near the melting point.

Experimental X-ray diffraction cannot capture signals from oxygen atoms clearly, as they are overwhelmed by signals from heavy La and $\mathrm{Zr}$ atoms. However, neutron diffraction will clearly capture oxygen atoms, and we plan to work on this aspect in the near future.

\subsubsection{Cation sublattice}

During MD simulation of solid-liquid coexistence, we observe a cation-disordered solid phase when the liquid portion of the supercell solidifies. Although this may serve as evidence for cation disordering, we cannot rule out the possibility that this disordered solid is metastable. When solidifying, the random chaotic liquid portion may not fully optimize and arrange itself to the thermodynamically most favored solid structure, due to the limited length of time (only on the order of 10 picoseconds). It requires a thermodynamic study on this disordered phase to assess its relative phase stability.

The energy of this phase is first extracted from coexistence MD simulations. 
Table 4: Pair correlation as an order parameter to quantify the randomness of newly formed solid from MD. The values for ordered pyrochlore and completely disordered random fluorite are listed for comparison.

$\begin{array}{cccc} & \text { Zr-Zr } & \text { La-La } & \text { Zr-La } \\ 2300 \mathrm{~K} & 0.36 \pm 0.01 & 0.36 \pm 0.01 & 0.27 \pm 0.02 \\ 2400 \mathrm{~K} & 0.31 \pm 0.02 & 0.32 \pm 0.02 & 0.37 \pm 0.02 \\ 2500 \mathrm{~K} & 0.32 \pm 0.02 & 0.32 \pm 0.02 & 0.35 \pm 0.04 \\ \text { pyrochlore } & 0.5 & 0.5 & 0 \\ \text { random fluorite } & 0.25 & 0.25 & 0.5\end{array}$

When solid-liquid coexistence evolves to pure solid phase, we find, in most cases, half of the supercell is in the cation-disordered phase, while the other half is in the original pyrochlore phase. The energy of this disordered phase is calculated to be $114 \mathrm{meV} /$ atom $\left(121 \mathrm{~kJ} / \mathrm{mol} \mathrm{La}_{2} \mathrm{Zr}_{2} \mathrm{O}_{7}\right)$, compared to fully ordered pyrochlore. This energy is much higher than the oxygen-sublattice disordered phase (33 meV/atom, $35 \mathrm{~kJ} / \mathrm{mol} \mathrm{La}_{2} \mathrm{Zr}_{2} \mathrm{O}_{7}$ ). Indeed, this penalty is so large that we find only the maximal configuration entropy could counteract it. At $2500 \mathrm{~K}$, the maximal configurational entropy is achieved if both cation and anion sublattices are completely random. Its contribution to free energy is

$$
-T S_{\text {fluorite }}=k T \sum n_{i} \ln x_{i} / \sum n_{i}=-113 \mathrm{meV} / \text { atom }
$$

Therefore, unless this cation-disordered phase is highly random (i.e., with virtually no short-range order) in reality, it would be thermodynamically unfavored with respect to the pyrochlore phase.

We use two approaches to determine the randomness of this disordered phase. Our analysis shows this phase is only partially disordered, so it is thermodynamically unstable. In order to quantify the extent of disordering, we introduce a specific pair correlation as an order parameter. We select a pair cluster twice the distance between nearest neighbor La-La, and we calculate the pair correlation of cations, as shown in Table 4 Two benchmarks, ordered pyrochlore and completely disordered random fluorite, are listed for comparison. These 


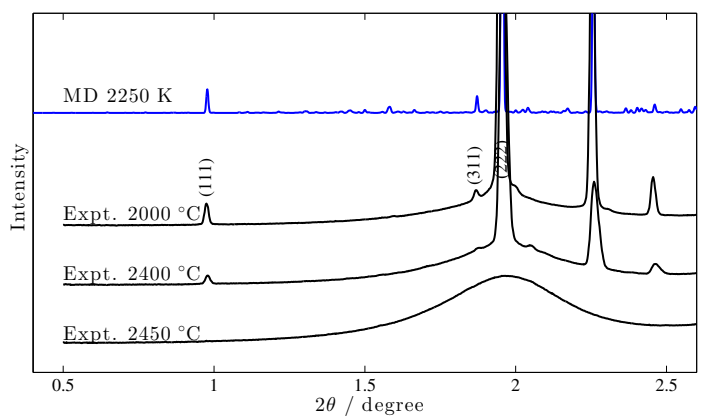

Figure 6: Synchrotron $(\lambda=0.10798 \AA)$ X-ray diffraction of $\mathrm{La}_{2} \mathrm{Zr}_{2} \mathrm{O}_{7}$ at $\sim 2000-2450{ }^{\circ} \mathrm{C}$. Pyrochlore (111) reflection is evident until complete melting. Theoretical X-ray diffraction from MD snapshots (generated by AFLOW/ACONVASP 73]) is plotted for comparision.

data suggest the newly formed solid is partially disordered. SQS analysis also shows the disordering is limited. We generate SQS assuming complete disordering on both sublattices, i.e., the (La, $\mathrm{Zr})$ and the (O, vacancy) sublattices. After structural optimization, we find some oxygen atoms moving to neighboring lattice sites, suggesting the SQS is unstable before configurational change. Such behavior means this disordered phase is constrained in a limited portion of configuration space, i.e., it is partially disordered.

Our theoretical result is confirmed by both X-ray diffraction and quenching experiments. Synchrotron X-ray diffraction experiments were performed on laser heated sample levitated in oxygen flow. Pyrochlore (111) reflections were observed up to complete melting (Fig. 6). The ratio of $(111) /(222)$ reflections was previously used for evaluation of radiation induced antisite disorder in pyrochlores 72. Our diffraction data indicate no significant variation (from 0.020 to 0.018$)$ in $(111) /(222)$ ratio with temperature. Experiments on containerless quenching and splat quenching of $\mathrm{La}_{2} \mathrm{Zr}_{2} \mathrm{O}_{7}$ melt were performed using a splittable nozzle aerodynamic levitator. The pyrochlore phase was observed in all experiments with $\mathrm{La}_{2} \mathrm{Zr}_{2} \mathrm{O}_{7}$, even though fluorite phase has been obtained by this method for $\mathrm{Eu}_{2} \mathrm{Zr}_{2} \mathrm{O}_{7}$ [74].

Differential thermal analysis also clearly indicates the absence of metastable phase. Heat effects on melting and solidifying are quantified by differential ther- 
mal analysis. As shown in Fig. 2, the values from integration of peaks in heat flow on melting and crystallization are consistent within experimental uncertainty, indicating no formation of a metastable phase of significantly different energy upon crystallization.

Although we have strong evidences from both computation and experiment, we note that we still cannot completely rule out the possibility of a cationdisordered phase. The fact that this phase is present in MD simulation indicates either the transition to pyrochlore phase is kinetically hindered or this phase is slightly unstable relative to the pyrochlore phase so MD simulation does not reflect the stability difference. X-ray diffraction results do not completely exclude the possibility of disordering either. As a thermal gradient is present in the diffracted volume [48, the disordered phase could occur $\sim 100{ }^{\circ} \mathrm{C}$ below melting. Even if this is the case, the possibility of transient defect fluorite phase occurrence in $\mathrm{La}_{2} \mathrm{Zr}_{2} \mathrm{O}_{7}$ right before melting can be treated more as a peculiarity of solid to liquid transition rather than solid state phase transition and of little significance for $\mathrm{La}_{2} \mathrm{Zr}_{2} \mathrm{O}_{7}$ application as a thermal barrier coating. Reported occurrences of lanthanum zirconate in disordered fluorite phase at low temperature in precipitates and in small particles from vapor deposition are likely to be induced by a surface energy term. This may play an important role in design of thermal barrier coating microstructures and choice of the method of their manufacturing and warrant separate investigation. However, analysis of these effects is beyond the scope of this paper.

\subsection{Heat capacity}

We calculate heat capacity in a temperature region up to the melting point. At each temperature, the heat capacity is calculated as derivative of enthalpy with respect to temperature. We run first-principles MD under the NPT ensemble to obtain the enthalpies at various temperatures. We include quantum corrections to vibrational energies, since they are significant at low tempera- 


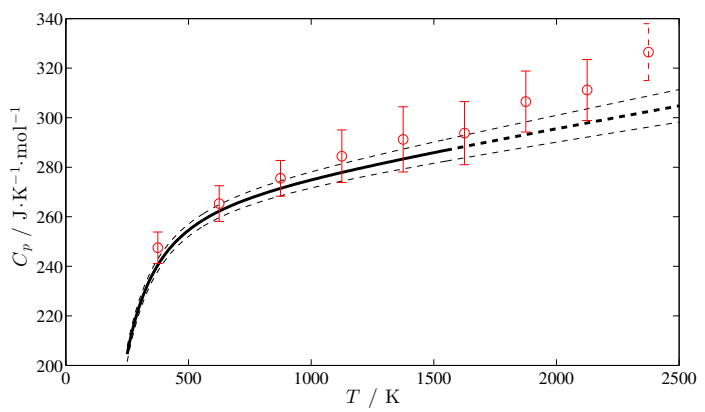

Figure 7: Heat capacity of pyrochlore $\mathrm{La}_{2} \mathrm{Zr}_{2} \mathrm{O}_{7}$ up to melting temperature. Computational results are shown in red. The last data point at $2375 \mathrm{~K}$ may be invalid due to the possible transition to oxygen-sublattice disordered phase near $2500 \mathrm{~K}$. No oxygen diffusion is observed below $2250 \mathrm{~K}$. The black curve is from experiment up to $1550 \mathrm{~K}$. 75

tures.

$$
\Delta H=\frac{1}{N} \sum_{i=1}^{3 N}\left(0.5+\frac{1}{\exp \left(h \nu_{i} / k_{B} T\right)-1}\right) \cdot h \nu_{i}-3 k_{B} T,
$$

320 The ATAT package [71] is employed to calculate the phonon frequencies. The calculated heat capacities are shown in red in Fig. 7 and are found in good agreement with available experimental data at lower temperature.

\subsection{Thermal expansion}

Using the same procedure, thermal expansion coefficients are calculated, as shown in Fig. 8. The calculated thermal expansion coefficient is $11.1 \times 10^{-6}$ $\mathrm{K}^{-1}$.

Experimental thermal expansion coefficient (Fig. 8) is refined for the range $1400-2100{ }^{\circ} \mathrm{C}$, from X-ray diffraction on a laser heated levitated sample. The difference in the experimental and calculated cell parameters is due to both possible deviation in stoichiometry in laser melted lanthanum zirconate, and the known overestimation from calculations (Table 1). However, the experimental value for thermal expansion coefficient, $11.5 \times 10^{-6} \mathrm{~K}^{-1}$, is in remarkable agreement with MD simulations. 


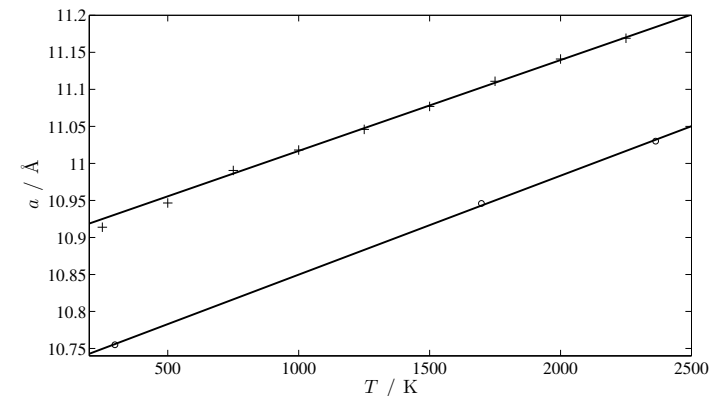

Figure 8: Lattice parameter at various temperatures in MD simulation $(+)$ and X-ray diffraction experiment (o). For theoretical thermal expansion, the coefficient is linearly fitted in temperature region $[1000,2250] \mathrm{K}$. Lattice parameter is subject to quantum effect at low temperature, thus the first two data points are underestimated. high temperature properties. Novel experimental techniques, such as structural studies at high temperature, provide validation between closely related phases 
often not unambiguously resolved computationally. Measurements of fusion en-

thalpies and heat capacities above $2000{ }^{\circ} \mathrm{C}$ are very challenging experimentally and can be greatly leveraged by computational methods.

\section{Acknowledgments}

This research was supported by ONR under grant N00014-12-1-0196 and by Brown University through the use of the facilities at its Center for Computation and Visualization. This work made use of the Extreme Science and Engineering Discovery Environment (XSEDE), which is supported by National Science Foundation grant number ACI-1053575. Use of the Advanced Photon Source (APS), an Office of Science User Facility operated for the DOE Office of Science by Argonne National Laboratory, was supported by the DOE under Contract No. DE-AC02-06CH11357.

\section{References}

[1] Labrincha JA, Frade JR, Marques FMB. J Mater Sci 1993;28:3809.

[2] Mitterdorfer A, Gauckler LJ. Solid State Ionics 1998;111:185.

[3] McCauley RA, Hummel FA. J Lumin 1973;6:105.

370 [4] Otaki H, Kido H, Hoshikawa T, Shimada M, Koizumi M. J Ceram Soc Jpn 1988;96:124.

[5] Andriamasinoro D, Kieffer R, Kiennemann A, Poix P. Appl Catal A-General 1993;106:201.

[6] Bussi J, Parodi S, Irigaray B, Kieffer R. Appl Catal A-General 1998;172:117.

375 [7] Chirayil TG, Paranthaman M, Beach DB, Lee DF, Goyal A, Williams RK, Cui X, Kroeger DM, Feenstra R, Verebelyi DT, Christen DK. Physica C 2000;336:63. 
[8] Knoth K, Huhne R, Oswald S, Schultz L, Holzapfel B. Acta Mater 2007;55:517.

380

[10] Miller RA. Surf Coat Tech 1987;30:1.

[11] Goward GW. Surf Coat Tech 1998;108:73.

[12] Evans AG, Mumm DR, Hutchinson JW, Meier GH, Pettit FS. Prog Mater Sci 2001;46:505.

385

[24] Hong Q-J, van de Walle A. J Chem Phys 2012;137:094114. 
[25] Belonoshko AB, Skorodumova NV, Rosengren A, Johansson B. Phys Rev B 2006;73:012201.

[26] Lin ST, Blanco M, Goddard WA. J Chem Phys 2003;119:11792.

${ }_{405}$ [27] Wang LG, van de Walle A. Phys Chem Chem Phys 2012;14:1529.

[28] Wang LG, van de Walle A, Alfe D. Phys Rev B 2011;84:092102.

[29] Hong Q-J, van de Walle A. J Chem Phys 2013;139:094114.

[30] Subramanian MA, Aravamudan G, Rao GVS. Prog Solid State Chem 1983;15:55.

${ }_{410}$ [31] Wilde PJ, Catlow CRA. Solid State Ionics 1998;112:173.

[32] Williford RE, Weber WJ. J Am Ceram Soc 1999;82:3266.

[33] Williford RE, Weber WJ, Devanathan R, Gale JD. J Electroceram 1999;3:409.

[34] Hohenberg P, Kohn W. Phys Rev B 1964;136:B864.

${ }_{415}$ [35] Kohn W, Sham LJ. Phys Rev B 1965;140:1133.

[36] Jones R, Gunnarsson O. Rev Mod Phys 1989;61:689.

[37] Kresse G, Furthmüller J. Comput Mater Sci 1996;6:15.

[38] Kresse G, Furthmüller J. Phys Rev B 1996;54:11169.

[39] Kresse G, Joubert D. Phys Rev B 1999;59:1758.

420 [40] Blöchl P. Phys Rev B 1994;50:17953.

[41] Perdew J, Burke K, Ernzerhof M. Phys Rev Lett 1996;77:3865.

[42] Nosé S. Mol Phys 1984;52:255.

[43] Nosé S. J Chem Phys 1984;81:511.

[44] Hoover WG. Phys Rev A 1985;31:1695. 
[45] Martyna GJ, Klein ML, Tuckerman M. J Chem Phys 1992;97:2635.

[46] Felice RA. AIP Conf Proc 2003;684[Pt. 2]:7116.

[47] Ushakov SV, Navrotsky A. J Mater Res 2011;26:845.

[48] Ushakov SV, Navrotsky A. J Am Ceram Soc 2012;95:1463.

[49] Toby BH, Von Dreele RB. J Appl Cryst 2013;46:544.

${ }_{430}$ [50] Heyd J, Scuseria GE, Ernzerhof M. J Chem Phys 2006;124:219906.

[51] Lakiza SM, Lopato LM. J Eur Ceram Soc 2005;25:1373.

[52] Zoz EI, Gavrish AM, Gulko NV. Izv Akad Nauk SSSR Neorg Mater 1978;14:109.

[53] Portnoi KI, Timofeeva NI, Salibekov SE, Romanovich IV. Izv Akad Nauk 435 SSSR Neorg Mater 1972;8:406.

[54] Rouanet A. Rev Int Hautes Temp 1971;8:161.

[55] Lin TH,Yu HC, Guisuanyan Xuebao 1964;3:159.

[56] Ewing RC, Weber WJ, Lian J. J Appl Phys 2004;95:5949.

[57] Lian J, Zu XT, Kutty KVG, Chen J, Wang LM, Ewing RC. Phys Rev B 440 2002;66:054108.

[58] Begg BD, Hess NJ, Weber WJ, Devanathan R, Icenhower JP, Thevuthasan S, McGrail BP. J Nucl Mater 2001;288:208.

[59] Sickafus KE, Minervini L, Grimes RW, Valdez JA, Ishimaru M, Li F, McClellan KJ, Hartmann T. Science 2000;289:748.

${ }_{445}$ [60] Minervini L, Grimes RW, Sickafus KE. J Am Ceram Soc 2000;83:1873.

[61] Chartier A, Meis C, Weber WJ, Corrales LR. Phys Rev B 2002;65:134116.

[62] Lian J, Wang L, Chen J, Sun K, Ewing RC, Farmer JM, Boatner LA. Acta Mater 2003;51:1493. 
[63] Hess NJ, Begg BD, Conradson SD, McCready DE, Gassman PL, Weber WJ. J Phys Chem B 2002;106:4663.

[64] Ushakov SV, Brown CE, Navrotsky A. J Mater Res 2004;19:693.

[65] Mills G, Jonsson H, Schenter GK. Surf Sci 1995;324:305.

[66] Henkelman G, Jonsson H. J Chem Phys 2000;113:9978.

[67] Sanchez JM, Ducastelle F, Gratias D. Physica A 1984;128:334.

${ }_{455}$ [68] van de Walle A, Ceder G. J Phase Equilib 2002;23:348.

[69] Zunger A, Wei SH, Ferreira LG, Bernard JE. Phys Rev Lett 1990;65:353.

[70] van de Walle A, Tiwary P, de Jong M, Olmsted DL, Asta M, Dick A, Shin

D, Wang Y, Chen LQ, Liu ZK. Calphad 2013;42:13.

[71] van de Walle A, Asta M, Ceder G. Calphad 2002;26:539.

${ }_{460}$ [72] Li YH, Uberuaga BP, Jiang C, Choudhury S, Valdez JA, Patel MK, Won J, Wang YQ, Tang M, Safarik DJ, Byler DD, McClellan KJ, Usov IO, Hartmann T, Baldinozzi G, Sickafus KE. Phys Rev Lett 2012;108:195504.

[73] Curtarolo S, Setyawan W, Hart GLW, Jahnatek M, Chepulskii RV, Taylor RH, Wanga SD, Xue JK, Yang KS, Levy O, Mehl MJ, Stokes HT, Demchenko DO, Morgan D. Comp Mater Sci 2012;58:218.

[74] Saradhi MP, Ushakov SV, Navrotsky A. RSC Advances 2012;2:3328.

[75] Sedmidubsky D, Benes O, Konings RJM. J Chem Thermodyn $2005 ; 37: 1098$. 